Imaginarios modernos sobre la comunicación para el desarrollo: una mirada desde el sur

Julio César Monasterio

Question, Vol. 1, N. 56, e002, octubre-diciembre 2017. ISSN 1669-6581

http://perio.unlp.edu.ar/ojs/index.php/question/article/view/4331

FPyCS | Universidad Nacional de La Plata

La Plata | Buenos Aires | Argentina

Recibido: 29-08-2017 Aceptado: 14-11-2017

Cita sugerida: Monasterio, J. (2017). Imaginarios modernos sobre la comunicación para el desarrollo: una mirada desde el sur. Question, 1(56), e002. doi: https://doi.org/10.24215/16696581e002

\title{
Imaginarios modernos sobre la comunicación para el desarrollo: una mirada desde el sur
}

\author{
Modern imaginaries on communication for development: a look from the south
}

\author{
Julio César Monasterio \\ Universidad Nacional del Comahue (Argentina) \\ juliomonasterio@yahoo.com.ar
}

\section{Resumen}

Este artículo centra su interés en el desentramado de las estrategias hegemónicas implementadas desde el norte global para la construcción de teorías y políticas relacionadas con la difusión de ideas, tecnologías, modelos, patrones y pautas de una cultura dadora, en materia de comunicación para el desarrollo. Bajo el sustento teórico de la Mass Communication Research, la fuerte presencia en nuestra región de los postulados de la Teoría de la Difusión de Innovaciones marcó desde la década del sesenta el camino a seguir por parte de las elites locales, respecto a las formas a partir de las cuales alcanzar el desarrollo. Este camino estaba ligado inexorablemente al impulso del crecimiento económico, a la exaltación de los sentires 
nacionalistas y al cambio de las actitudes tradicionales y arcaicas a favor de la instrumentalización del progreso y la técnica.

Proponemos aquí llevar adelante una mirada desde una comunicología del sur, que entienda al sur, en los términos de Santos (2009) en tanto locus de enunciación privilegiado, desde el plano geográfico como desde el geopolítico, a partir del cual pensar la realidad histórica de los pueblos oprimidos por parte de la impronta colonial iniciada con la modernidad capitalista europea, así como a las derivas y nuevas modalidades que asume dicha impronta en nuestro presente.

Palabras clave: comunicación; desarrollo; Modernidad; colonialidad; sur global.

\section{Abstract}

This article focuses its interest on the unraveling of the hegemonic strategies implemented from the global north for the construction of theories and policies related to the diffusion of ideas, technologies, models, patterns and patterns of a donor culture, in the matter of Communication for the development. Under the theoretical support of Mass Communication Research, the strong presence in our region of the postulates of the Theory of the Diffusion of Innovations marked since the decade of the 60's the way to follow by the Local elites with respect to the ways in which development can be achieved. This path was inexorably linked to the impulse of economic growth, to the exaltation of nationalist sentiments, and to the change of traditional and archaic attitudes in favor of the instrumentalisation of progress and technique.

We propose here to take a look from a Southern Communication, which understands the South, in the terms of Santos (2009) as a locus of privileged enunciation, from the geographic and geopolitical level, from which to think reality Historical of the oppressed peoples by the colonial imprint initiated with the European capitalist modernity, as well as to the drifts and new modalities that assume this imprint in our present.

Keywords: communication; development; Modernity; coloniality; global south.

El presente escrito se propone desarrollar un análisis referido a la relación entre construcción de conocimientos y política en América Latina para, posteriormente, problematizar las concepciones de desarrollo surgidas luego de la Segunda Guerra Mundial, desde una mirada que busque reconocer las transformaciones históricas y el enfoque de lo complejo, con el objetivo de dar cuenta de sus implicancias en el campo de la comunicación y la cultura. Nos 
propondremos, también, recuperar algunos de los principales aportes realizados desde nuestro continente que surgen como alternativas críticas a las teorías de la modernización.

El concepto de desarrollo y, más precisamente, el de comunicación para el desarrollo será el que funcionará como eje transversal del presente trabajo. Por esto nos parece sustancial, en un primer momento, recuperar algunas de las estrategias discursivas hegemónicas del proyecto moderno/colonial para la construcción de conocimientos a partir de la generación de un pensamiento binario y oposicional. En este sentido, la puesta en tensión de imaginarios modernos nos permitirá apreciar su materialidad adquirida en políticas específicas y en instituciones que los sostienen.

Posteriormente, nos centraremos en la revisión de las estrategias comunicacionales de las Teorías de la Modernización, a partir de la implementación de políticas de difusión de innovaciones y de la preocupación, por parte de los centros hegemónicos de producción de conocimiento, por la compatibilización entre desarrollo y subdesarrollo y por la ruptura de las "formas tradicionales" de nuestro continente.

Para finalizar, intentaremos demostrar cómo el pensamiento comunicacional latinoamericano nos propone otras claves para entender la relación entre comunicación y desarrollo en sintonía con lo planteado por los estudios sociales de la dependencia, materializados en lo que popularmente se conoce como Teoría de la Dependencia, los cuales buscaron romper con el paradigma dominante de la modernización; un paradigma que entendía a la comunicación como un sistema unidireccional, lineal y vertical y modificarlo por otro bidireccional, horizontal, comprometido y popular.

\section{La construcción de un pensamiento oposicional}

Los modos de construcción de realidad generados a partir de un pensamiento binario otorgaron centralidad a diferentes tipos de sujetos, de prácticas, de espacios y de saberes. Uno de los principales binarismos instaurados por la modernidad ha sido la disociación entre el hombre y la naturaleza, entre razón y mundo, disociación que refuerza un proyecto ético, político y filosófico de la modernidad eurocentrada y que rompe con la visión orgánica del mundo. En palabras de Lander:

la ruptura ontológica entre la razón y el mundo quiere decir que el mundo ya no es un orden significativo, está expresamente muerto (...) El mundo se convirtió en lo que es para los ciudadanos el mundo moderno, un mecanismo desespiritualizado que puede ser captado por los conceptos y representaciones construidos por la razón (2000: 15). 
En este sentido, el proyecto moderno colonial estableció jerarquías referidas a la construcción de una determinada forma de conocimiento, el conocimiento científico, por sobre otros saberes, y de la teoría por sobre la práctica; la cartografía moderna constituyó ciertas regiones como centros -Europa, principalmente los países de la Europa Occidental y Estados Unidos- y puso en situación de periferia al resto -el sur de Asia, África, América Latina y el Caribe-; estableció criterios de jerarquización y categorización raciales -blanco/negro-, de género -varón/mujer-, culturales -cultura/naturaleza, cultura de elite/culturas populares- (Monasterio, 2014).

La formación de un pensamiento oposicional asociado a las construcciones de imaginarios y representaciones mentales tuvo su materialidad concreta en políticas y en instituciones de carácter disciplinario, tales han sido las escuelas, las cárceles, las leyes y las ciencias sociales, entre otras, que posibilitaron una vinculación específica entre conocimiento y disciplina (CastroGómez, 2000). El proyecto moderno/colonial llevó adelante estas estrategias mencionadas, a partir de las cuales comprender los procesos de construcción de sociabilidades y subjetividades e históricamente ha buscado correr al sujeto de la investigación con la pretensión de otorgar neutralidad y objetividad a la producción del conocimiento.

Consideramos a esta acción de búsqueda de corrimiento del sujeto cognoscente como un acto sustancial del proceso de "despolitización de la ciencia" y de su intento por ocultar las modalidades, las formas y las condiciones a partir de las cuales se lleva adelante esta tarea, pretendiendo, siguiendo a Castro-Gómez, "hacerse un punto de vista sobre todos los puntos de vista, pero sin que de ese punto de vista pueda tenerse un punto de vista" (2008: 123).

Visibilizar esta estrategia es poner de manifiesto el "carácter provinciano" de las ciencias sociales y su pretensión universalizante, ya que el ocultamiento de estas condiciones plantea una trama histórica que constituye a un sur entendido como periferialidad. El norte hegemónico y el sur periférico y marginal (he aquí otra construcción discursiva oposicional) no se pueden comprender por fuera del lugar de las

prácticas cognitivas de las clases, de los pueblos y de los grupos sociales que han sido históricamente victimizados, explotados y oprimidos, por el colonialismo global y presentes hoy en el marco de la colonialidad global. El Sur es, pues, usado aquí como metáfora del sufrimiento humano sistemáticamente causado por el colonialismo y el capitalismo (De Sousa Santos, 2009: 12).

Se trata, entonces, de dar cuenta cuáles han sido las condiciones que posicionaron a este sur como periférico, reconociendo que esta dimensión no es sólo geográfica sino también, y principalmente, epistémica.

El sur atrasado, tradicional y arcaico se constituyó en uno de los grandes obstáculos a superar por parte del proyecto modernizador de occidente de los siglos XIX y XX. La noción de progreso se forjó como uno de los principales lineamientos modernos en oposición al atraso de 
los países del denominado tercer mundo (grandes regiones de América Latina, África y Asia). Fue aquí muy importante el rol ocupado por las elites locales para impulsar y materializar estos postulados con la finalidad de "civilizar a los bárbaros". Retomando el planteo de CastroGómez, "una de las tareas centrales de las ciencias del hombre es mostrar (...) que no todas las poblaciones del planeta se encuentran en el mismo nivel de la evolución humana y que esta asimetría obedece a un plan maestro de la naturaleza" (2008: 135).

Cabe destacar que este proceso civilizatorio se llevó a cabo, bajo distintas modalidades, en las diferentes regiones subalternizadas de América Latina. Podemos mencionar como ejemplos, entre muchos otros, al proceso desarrollado durante el siglo XIX en la Patagonia argentina denominado "Conquista del Desierto", que fue legitimada por los principales exponentes del pensamiento latinoamericano de la época a partir de la negación ontológica de los sujetos pertenecientes a esa región, a los dispositivos de blancura desarrollados en Colombia gracias a tecnologías de gobierno pensadas en función de una "limpieza de sangre de la raza colombiana" a partir de las políticas de inmigración y mestizaje (1) y al rol de la Iglesia para, desde un modelo de la cristiandad, llevar adelante su campaña de evangelización en América Latina.

No es intención del presente trabajo desarrollar y llevar adelante un análisis de cada uno de los diferentes procesos civilizatorios de poblaciones en América Latina, pero sí marcar que existe una continuidad y una linealidad en las políticas de los Estados latinoamericanos, que se sustenta en una visión jerarquizadora de las poblaciones y de sus prácticas que, al día de hoy, continúa bajo nuevas modalidades.

\section{Comunicación y desarrollo. Estrategias hegemónicas para la difusión de innovaciones}

Los cambios sociales que se desplegaron luego de la Segunda Guerra Mundial pusieron en evidencia nuevas formas de comprender el mundo, a partir de la imagen propuesta por las Teorías de la Modernización y la imposición de la idea de desarrollo. Estas nuevas maneras de mirar la realidad pusieron su foco en diferentes cuestiones, entre las que podemos destacar, según Scribano (2004), la visión de sujeto, sus recursos de diferenciación, su horizonte de comprensión y la noción espacio temporal.

En el campo de la comunicación y la cultura, una de las propuestas teóricas inscriptas dentro de las Teorías de la Modernización, que contó con mayor aceptación a nivel global, fue la Teoría de la Difusión de Innovaciones. Esta teoría, que tuvo entre sus principales exponentes a los sociólogos Everett Rogers, Daniel Lerner y Elihu Katz y al comunicador Wilbur Schramm, se sustentó en los principios del funcionalismo norteamericano y de la psicología conductista, entendiendo que la comunicación masiva es un factor de modernización (desarrollo) para las 
sociedades subdesarrolladas a partir de la difusión de tecnologías, modelos, patrones y pautas de una cultura dadora para que sean aceptadas, incorporadas e internalizadas por parte de las culturas destinatarias.

Los principales objetivos de la Teoría de la Difusión se centraban en que los países subdesarrollados puedan alcanzar el estadio del desarrollo a partir del impulso del crecimiento económico, de la exaltación de un sentir nacional y del cambio de las actitudes tradicionales y arcaicas a favor de la instrumentalización del progreso y la técnica. El desarrollo aparece como un concepto unificador en el lenguaje de las relaciones globales luego de la Segunda Guerra Mundial, esparcido desde las sociedades industrializadas hacia los centros urbanos en los países periféricos, y de estas áreas a las "atrasadas zonas rurales". Desarrollo será equivalente a progreso, bienestar y prosperidad legitimando una visión etnocéntrica del subdesarrollo.

El difusionismo se convirtió entonces en el paradigma dominante respecto de la cuestión del desarrollo, estableciendo un entramado geopolítico explicado a partir de patrones que asociaron el estado de los países subdesarrollados a factores ligados a la tradición, al carácter y la cultura de los pueblos, factores que limitaban sus posibilidades de crecimiento. Desde esta concepción, la comunicación para el desarrollo funciona como un instrumento para la incorporación de los países en "vías de desarrollo" dentro del sistema comunicativo mundial para la difusión de la tecnología industrial, de las instituciones sociales modernas y del modelo de sociedad de libre mercado.

Las teorías desarrollistas son teorías que apuntan, principalmente, a la búsqueda del cambio social. En este punto cabría preguntarnos cómo se convierte, entonces, la comunicación como agente de cambio. Podemos decir que, a partir de una primera aproximación, lo hacen a través de las modernas técnicas de persuasión, difusión e información con las que cuentan los medios masivos, con la finalidad de imponer valores universales como los de progreso, movilidad social, individualismo y consumo. $\mathrm{Y}$ en la búsqueda del pasaje de lo tradicional a lo moderno, se intentará que esa transición se lleve a cabo bajo un marco institucional carente de conflicto.

Es imposible desconocer el peso que han tenido las políticas de comunicación para el desarrollo en la región y que se materializaron fuertemente en tres ámbitos de la vida social: la planificación familiar, la difusión de innovaciones en el campo (también llamada "revolución verde") y la implementación de nuevas tecnologías en el ámbito educativo (2).

Estas políticas formaron parte de las estrategias de la Alianza para el Progreso que, a partir del apoyo económico del Banco Interamericano de Desarrollo y de otras organizaciones privadas y del apoyo político de organizaciones internacionales (UNESCO, PNUD, OEA, entre otras), garantizaron la presencia de Estados Unidos en las políticas económicas y socioculturales de América Latina (3). 


\section{Dependencia y liberación. Algunas claves para mirar la "comunicación democrática para el desarrollo"}

Uno de los grandes problemas de las teorías de la modernización es que depositó toda responsabilidad sobre el cambio social en los actores individuales (Scribano, 2004) y no en los procesos de "configuraciones culturales" latinoamericanas (4). Además, fue incapaz de explicar cómo se llevaron adelante los procesos de cambio en un entramado de relaciones sociales tan complejo como lo es el de América Latina. Por esto, cabe destacar que en nuestro continente los intentos de implementación de estas políticas no estuvieron exentos de una importante cantidad de luchas sociales, políticas y culturales y que los intentos de modernización en América Latina se sustentaron a partir de una serie de tesis equivocadas e incuestionables. Las mismas son: 1. los países latinoamericanos son sociedades duales; 2. el progreso en América Latina se realizaría mediante la difusión de los productos del industrialismo a las zonas atrasadas, arcaicas y tradicionales; 3 . la existencia de estas zonas es un obstáculo para la formación del mercado interno y para el desarrollo del capitalismo nacional y progresista; 4. la burguesía nacional tiene interés de romper el poder y el dominio de la oligarquía terrateniente; 5. el desarrollo en América Latina es creación y obra de una clase media nacionalista, progresista, emprendedora y dinámica, y el objetivo de la política social y económica de nuestros gobiernos debe ser estimular la "movilidad social" y el desarrollo de esta clase; 6 . la integración nacional de América Latina es producto del mestizaje y 7. el progreso en América Latina sólo se realizará mediante una alianza entre los obreros y los campesinos, alianza que impone la identidad de intereses de estas dos clases (Stavenhagen, 1981).

¿Por qué se produce, entonces, en América Latina una nueva manera de pensar los procesos de desarrollo y, más específicamente para el tema que nos convoca, de Comunicación para el desarrollo? En principio podemos destacar tres aspectos que se encuentran en íntima relación. A saber:

a. Contexto social, político y económico. A modo de ejemplo, se sucedieron importantes revoluciones populares, principalmente en Cuba y Chile, que pusieron en tensión los principales postulados del sistema capitalista; en materia de comunicación la propuesta del Nuevo Orden Informativo Internacional (NOII) y las Políticas Nacionales de Comunicación (PNC) que encuentran momento más importante en el Informe Mc Bride con la propuesta de un Nuevo Orden Mundial de la Información y la Comunicación (NOMIC).

b. Nuevas alternativas teóricas. Estudios sociales de la dependencia, críticos de los ideales y de las políticas del desarrollismo y de la modernización, que plantean que la constitución de los países periféricos no sólo se da por una dependencia económica, sino también por las implicancias de lo político y de lo cultural. Aquí rescataremos la propuesta de Cardoso y Faletto 
(1977) para comprender las relaciones entre centros y periferias y el rol que tuvieron las elites criollas para su afianzamiento.

c. Surgimiento de prácticas alternativas de comunicación, que devienen con la adopción de los estados de América Latina a las teorías modernizadoras y con el avance de las dictaduras militares en la región.

La investigación en comunicación y cultura(s) en América Latina tiene bastos antecedentes; sin embargo, podemos tomar como punto de partida la obra realizada en 1963 por Antonio Pasquali, denominada Comunicación y Cultura de masas, ya que da inicio a una cantidad de abordajes críticos desde América Latina en materia comunicacional y que surgieron como respuesta a la estrategia instrumental de la Comunicación para el desarrollo. El proyecto democratizador comunicacional impulsado por una importante cantidad de países del tercer mundo tiene como antecedente central la Conferencia Intergubernamental de Comunicación (Costa Rica, 1976), que permite proyectar los lineamientos de las PNC y del NOMIC.

Sin embargo, las pujas entre los esfuerzos por la búsqueda de una comunicación democrática y el ascenso de los gobiernos dictatoriales, durante la década del setenta, terminaron modificando el mapa comunicacional de las décadas siguientes. En líneas generales, el corrimiento del Estado dio lugar a la necesidad de una fuerte organización en materia de comunicación popular y comunitaria.

En el momento de, quizás, mayor influencia de la aplicación instrumental de la comunicación para el desarrollo, Luis Ramiro Beltrán (2005) propone en 1973, trastocar la concepción del desarrollo y entenderlo como

un proceso dirigido de profundo y acelerado cambio sociopolítico que genere transformaciones sustanciales en la economía, la ecología y la cultura de un país a fin de favorecer el avance moral y material de la mayoría de la población del mismo en condiciones de dignidad, justicia y libertad (15).

Es decir, Beltrán entiende la periferialidad de la región latinoamericana y menciona las condiciones que generaron esta condición. Pero, al mismo tiempo, también destaca su potencialidad libertaria. Así, en lo que a este trabajo respecta, a los movimientos de la Filosofía de la liberación, la Teología de la liberación, la Antropología de la liberación y la Pedagogía de la liberación, deberíamos sumarle la propuesta por una Comunicología de la liberación, en la que la que autores como el propio Beltrán, Pasquali, Schmucler, Marques de Melo y Díaz Bordenave -entre otros- propusieron una alternativa a la concepción hegemónica de los procesos comunicacionales. Quizás uno de los desafíos para pensar los procesos de comunicación y desarrollo está centrado en continuar con estas discusiones, entendiendo "las 
tensiones con las que convive el desarrollo frente a las postulaciones de órdenes encontrados, muchas veces de sentidos irreconciliables" (Cimadevilla, 2015: 413).

\section{Algunas consideraciones finales}

El borramiento de prácticas de socialización y la imposición de nuevos patrones socioculturales son una constante que se repite a lo largo de la historia en la operatoria ideológica y política del sistema capitalista/moderno. Podemos decir que la construcción de lo no-occidental, del "otro cultural", se realiza a partir de una serie de gestos que van delineando a ciertas prácticas culturales como deficientes, disfuncionales, atrasadas e incompatibles con los ideales modernos de civilización, progreso y desarrollo. De esta manera, los pueblos originarios, las culturas populares y los sectores subalternizados fueron nombrados, categorizados, invisibilizados $y$, posteriormente, visibilizados negativamente, a partir de operaciones metafóricas de exotización, folclorización, racialización, infantilización y animalización, que legitimaron la superioridad occidental.

Problematizar los lugares desde los cuales se constituyeron los sustentos teóricos y filosóficos modernos que justificaron una forma de ver el mundo fue uno de los objetivos del presente trabajo, entendiendo que toda teoría es situacional y que no se puede comprender por fuera de su locus de enunciación y que, además, toda representación de la realidad es, al mismo tiempo, una forma de intervención y de influencia sobre la misma.

Las respuestas del pensamiento latinoamericano a las perspectivas instrumentales de las teorías desarrollistas apuntaron a la construcción de una alternativa crítica a la comunicación para el desarrollo. En este sentido, sus principales aportes intentaron romper con las tesis equivocadas sobre el desarrollo, planteadas por Stavenhagen en 1981, reforzando el carácter local de las prácticas e historias en aras de proponer un pensamiento y prácticas comunicacionales alternativas.

Repensar las modalidades a partir de las cuales se llevaron adelante las estrategias de difusión del desarrollo en nuestro continente, de sus respuestas críticas y de su vigencia en la actualidad, nos interpela en relación a una tarea que implica una descolonización de las ciencias sociales y una salida del estado de sujeción colonial (Scribano, 2012). Y este desafío solo será posible si avanzamos en la construcción de conceptualizaciones latinoamericanas que se centren posiciones de estructuras duales y puedan dar cuenta de la complejidad del entramado social, cultural, político y económico de nuestra América.

Para finalizar, cabe destacar que una Comunicología del sur debe poner en tensión las formas canónicas y tradicionales a partir de las cuales se desarrolla la producción del conocimiento en el campo, posibilitando y favoreciendo la irrupción de epistemologías otras a través de un 
diálogo horizontal de prácticas, de seres y de saberes marginados y estigmatizados. Para esto, es fundamental que la academia pueda cuestionar(se) su locus de enunciación privilegiado, reconociendo que es resultado de las formaciones sociales de la historia capitalista, moderna/colonial.

Una Comunicología del sur debe promover la producción de abordajes críticos, que no sólo se limiten a una mera tarea descriptiva y celebratoria de las innovaciones técnicas sino que, principalmente, apunten a una indagación y a una interpelación de las causas que generaron históricamente las desigualdades estructurales en nuestra región y a la relación de estas con los procesos comunicacionales y culturales. Tomar la opción de posicionarse desde el sur supone, en definitiva, la necesidad de disputar agendas y de construir marcos referenciales propios que rompan con la extranjería a la que nos enfrentamos cuando pensamos nuestros procesos sociales.

\section{Notas}

(1) Cfr. Castro-Gómez, S. (2009). Tejidos oníricos: movilidad, capitalismo y biopolítica en Bogotá, 1910-1930. Bogotá: Editorial Pontificia Universidad Javeriana.

(2) Para poder profundizar sobre estos tres ejes: cfr. Pereira G., José Miguel; Bonilla V., Jorge Iván; Benavides C., Julio Eduardo (1998). La comunicación en contextos de desarrollo: Balances y perspectivas. Signo y Pensamiento, 32, Universidad Javeriana, pp. 119-138.

(3) Hacia finales de la década del cincuenta, en un contexto geopolítico marcado por el apogeo de la Guerra Fría, las estrategias capitalistas de expansión se centraron en la difusión de políticas y programas "racionales" desde los centros hacia las periferias. La Alianza para el Progreso fue un "proyecto continental del presidente John F. Kennedy que prometía una revolución en libertad (frente al peligro de la insurgencia revolucionaria de los sesenta y la reciente revolución cubana en particular)" (Huergo, 2005: 9). Frente a este clima de efervescencia social que se vivió en América Latina durante esos años, Estados Unidos optó por tener una fuerte presencia territorial en América Latina.

(4) Este concepto es tomado de Grimson, Alejandro (2011). Los límites de la cultura. Crítica de las teorías de la identidad. Buenos Aires: Siglo XXI. De manera muy resumida, la noción de configuración cultural nos propone desencajar a la cultura de su supuesta autonomía, para pensarla como una dimensión transversal y de interrelación permanente con lo político, lo histórico, lo económico y lo social, escenarios dónde la desigualdad y el poder están siempre presentes. 


\section{Bibliografía}

Beltrán Salmón, L. R. (2005). La comunicación para el desarrollo en Latinoamérica: un recuento de medio siglo. III Congreso Panamericano de la Comunicación, Universidad de Buenos Aires, Buenos Aires.

Cardoso, F. y Faletto, E. (1977). Dependencia y Desarrollo en América Latina. Buenos Aires: Siglo XXI.

Castro-Gómez, S. (2000). Ciencias sociales, violencia epistémica y el problema de la 'invención' del otro. En Lander, E. (Comp.). La colonialidad del saber: eurocentrismo y ciencias sociales. Perspectivas latinoamericanas (pp. 285-303). Buenos Aires: CLACSO.

Castro-Gómez, S. (2008). El lado oscuro de la 'época clásica'. Filosofía, ilustración y colonialidad en el siglo XVIII. En VV.AA. El color de la razón: racismo epistemológico y razón imperial (pp. 119-152). Buenos Aires: Ed. Del Signo.

Cimadevilla, G. (2015). Trayectos aplicados y académicos de la convergencia comunicacióndesarrollo. En Bolaño, C. y otros (coords.). La contribución de América Latina al campo de la comunicación. Historia, enfoques teóricos, epistemológicos y tendencias de la investigación (pp. 399-418). Buenos Aires: Prometeo Libros.

Grimson, A. (2011). Los límites de la cultura. Crítica de las teorías de la identidad. Buenos Aires: Siglo XXI.

Huergo, J. (2005). Desafíos de la extensión desde una perspectiva sociocultural. Revista Dialoguemos. Instituto Nacional de Tecnología Agropecuaria (INTA), Argentina.

Lander, E. (2000). Ciencias sociales: saberes coloniales y eurocéntricos. En Lander, E. (Comp.) La colonialidad del saber: eurocentrismo y ciencias sociales. Perspectivas latinoamericanas. Buenos Aires: CLACSO.

Monasterio, J. (2014). Invenciones modernas y recorridos de investigación. Astrolabio, 13, 124145.

Pereira, J., Bonilla, J. y Benavides, J. (1998). La comunicación en contextos de desarrollo: Balances y perspectivas. Signo y Pensamiento, 32, pp. 119-138.

De Sousa Santos, B. (2009). Una epistemología del sur La reinvención del conocimiento y la emancipación social. México: CLACSO-Siglo XXI.

Scribano, A. (2004). Combatiendo fantasmas. Teoría Social Latinoamericana, una Visión desde la Historia, la Sociología y la Filosofía de la Ciencia. Santiago de Chile: Ediciones MAD Universidad de Chile.

Scribano, A. (2012). Teorías Sociales del Sur: una mirada post-independentista. Córdoba: Estudios Sociológicos Editora. 
Shohat, E. y Stam, R. (2002). Multiculturalismo, cine y medios de comunicación. Crítica del pensamiento eurocéntrico. Barcelona: Paidós.

Stavenhagen, R. (1981). Sociología y Subdesarrollo. México: Nuestro Tiempo.

Torrico Villanueva, E. (2009). Luis Ramiro Beltrán: por una comunicología de la liberación. Chasqui. Revista latinoamericana de comunicación, 105, pp. 8-13. 